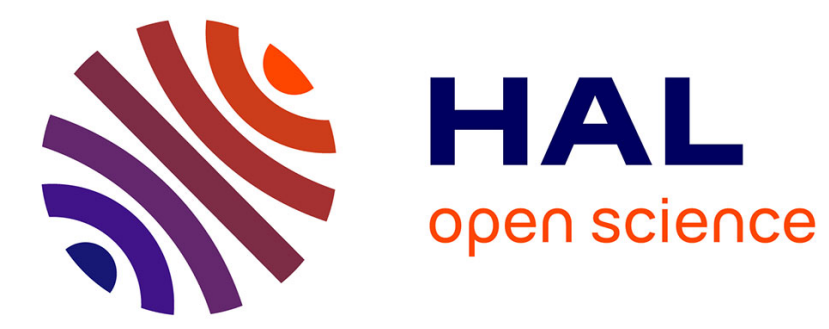

\title{
Les villes des haras nationaux
}

Damien Bruneau

\section{To cite this version:}

Damien Bruneau. Les villes des haras nationaux. Espaces et sociétés (Paris, France), 2017, Ces villes dont on ne parle pas, 168-169, pp.89-108. 10.3917/esp.168.0089 . hal-03320245

\section{HAL Id: hal-03320245 \\ https://hal.science/hal-03320245}

Submitted on 18 Aug 2021

HAL is a multi-disciplinary open access archive for the deposit and dissemination of scientific research documents, whether they are published or not. The documents may come from teaching and research institutions in France or abroad, or from public or private research centers.
L'archive ouverte pluridisciplinaire HAL, est destinée au dépôt et à la diffusion de documents scientifiques de niveau recherche, publiés ou non, émanant des établissements d'enseignement et de recherche français ou étrangers, des laboratoires publics ou privés. 
Damien BRUNEAU

Agrégé de géographie

Lycée Félix Le Dantec

Rue des Cordiers

22300 Lannion 


\section{Les villes des haras nationaux}

L'implantation des haras nationaux dans telle ou telle ville révèle le poids des régions d'élevage ainsi que celui de l'Histoire. Le choix récurrent d'une ville centrale et d'un emplacement prestigieux dans son cœur est à mettre en lien avec d'autres services publics. Cependant, il s'agit surtout de petites et moyennes villes d'autant plus touchées par la réforme de l'État depuis les années 2000. Dès lors, les trajectoires des différentes villes des haras nationaux sont très variables : fermeture précoce, fermeture attendue, reprise par une collectivité territoriale ou, au contraire, renforcement d'un pôle national. Ce devenir dépend de l'enracinement de la fonction équine, du patrimoine immobilier, de la mobilisation locale et des stratégies de développement urbain.

Haras - Implantation - Patrimoine - Réforme - Villes

National stud farms' cities

The implementation of national stud farms in some French cities reveals the weight of breeding areas as well as of history. The recurrent selection of a central city and a prestigious location in its heart is to be connected with other public services. However, it is mainly small and medium cities particularly affected by the government's reforms since the 2000s. Therefore, the trajectories of different national stud farms' cities are highly variable: early closure, expected closure, recovery by a local authority or, on the contrary, strengthening a national center. This fate dependent on the entrechment of equine function, local mobilization and urban development strategies.

Cities - Heritage - Implementation - Reforms - Stud farms

Las ciudades de las nacionales yeguadas

La ubicación de las nacionales yeguadas en algunas ciudades revela el peso de las zonas crias y el de la historia. La elección de una ciudad central y una localización privilegiada en su corazón es recurrente en relación con otros servicios públicos. No obstante, es principalmente pequeñas y medianas ciudades especialmente afectadas por la reforma del Estado desde la década de 2000. Por lo tanto, las trayectorias de diferentes ciudades de las nacionales yeguadas son muy variables : cierre anticapado, cierre esperado, tomada por una autoridad local o, por el contrario, el fortalecimiento de un centro nacional. Esto destino depende del afianzamiento de la función equina, la movilización local y las estrategias de desarrollo urbano.

Ciudades - Patrimonio - Reforma - Ubicación - Yeguadas 
Haras royaux, haras impériaux, haras nationaux... : quel que soit le régime politique, guider l'évolution des races équines et aider la production pour satisfaire les besoins en chevaux de toutes sortes est une constance de la France. Une administration longtemps restée spécifique gère ces établissements où sont entretenus des étalons reproducteurs sélectionnés, mais aussi des juments et des poulains, afin de préserver, multiplier et améliorer les races équines. De fait, nous avons affaire à une politique multiséculaire de soutien de l'État à la filière (Mulliez, 2004) : un service public avéré qui a affecté de manière variable une vingtaine de villes depuis au moins un siècle et demi avec la loi Bocher de 1874. Les haras nationaux, nommés de la sorte par commodité, même quand il s'agit d'un simple dépôt d'étalons sans jument ni poulain comme à Annecy (Kinossian, 2012), forment un réseau de villes complété notamment par des dizaines de stations de monte. Celles-ci voient certains fonctionnaires d'un haras national se décentraliser avec plusieurs étalons de races différentes pendant trois ou quatre mois, le plus souvent de février à juillet, pour réaliser les saillies dans ces sites secondaires, appelés par la suite centres techniques.

Cette fonction de service dans la politique du cheval en France donne à certaines villes un rayonnement sur de vastes circonscriptions. Celles-ci ont été pendant longtemps déterminées par les berceaux des races équines afin de répondre aux besoins de l'armée française, des agriculteurs et des éleveurs, puis sur un découpage purement administratif à partir des années 1970. Cette étude concerne les 23 haras nationaux qui existaient sur la période allant de 1974 à 2004. Ils sont implantés majoritairement en ville dans un spectre démographique très large allant de la petite ville, Montier-en-Der par exemple, à la métropole strasbourgeoise en passant par de nombreuses villes de taille moyenne. Selon l'INSEE, 8 villes des haras nationaux accueillent une population municipale entre 2000 et 20000 habitants et 11 sont comprises entre 20000 et 200000 habitants (tableau). Ces villes se définissent donc en creux: elles ne sont ni des capitales régionales aux fonctions métropolitaines (hormis Strasbourg $^{1}$ ) ni des petites villes cantonnées à une desserte locale (Santamaria, 2000). Les séculaires haras nationaux du Pin et de Pompadour ont ainsi un rayonnement majeur qui dépasse largement cet horizon. Ces villes sont avant tout des petites et moyennes villes qui regroupent aujourd'hui près du quart de la population française selon l'INSEE (Floch, 2011) et sur qui l'Histoire fait peser une fonction équestre plus ou moins dimensionnée pour elles. D'ailleurs, cette fonction est intégrée symboliquement dans le logo de quatre communes accueillant un haras national (Hennebont, Les Bréviaires, Pau-Gelos et Saint--Lô), une incontestable identification de la ville à ce service public mais aussi à une activité économique spécifique.

Depuis les années 2000, l'État a justement réduit les missions de service public de ces haras nationaux au profit des collectivités territoriales et de l'étalonnage privé. Il s'agirait à la fois de s'adapter aux nouvelles exigences des cavaliers et des passionnés de chevaux mais aussi au coût financier de ces activités. Cette réorganisation ne va pas sans influencer économiquement, socialement et financièrement les villes accueillant ces haras nationaux. De fait, il s'agit de se demander, dans une logique multiscalaire, ce qui a déterminé l'implantation des haras nationaux dans certaines villes plutôt que d'autres et en quoi le devenir des haras nationaux et de leurs bâtiments est un enjeu local majeur. Pour apporter des éclairages sur ces villes souvent méconnues, nous aborderons tout d'abord le rôle du passé et de la centralité dans la localisation générale des villes des haras nationaux. Puis, il sera question des effets sur ces villes de la volonté forte et récente de réformer l'État ainsi que l'émergence des nouveaux usages de ces lieux marqués durablement par la fonction équine.

\section{Les choix géohistoriques des villes d'implantation des haras nationaux}

$1 \quad$ La ville de Besançon est considérée comme une ville moyenne par la taille même si elle est une capitale régionale (Santamaria, 2012). 
Depuis deux siècles, le choix d'implanter un haras national est intimement lié au découpage, plus ou moins rationnel, des circonscriptions.

Dans un premier temps, un décret impérial de 1806 organise le territoire en 6 arrondissements (Nord, Ouest, Centre, Midi, Est et Nord-Est) pour 6 haras centralisateurs (respectivement Le Pin-au-Haras, Langonnet, Pompadour, Pau-Gelos, Turin et Zweibrücken) même s'ils sont loin d'être des lieux centraux de leur circonscription. Ceci s'explique à la fois par l'héritage royal (Le Pin-au-Haras, Pompadour) et les créations liées aux extensions révolutionnaires du territoire français (Turin dans le Piémont, Zweibrücken en Rhénanie). Cette répartition n'est alors que l'esquisse mouvante d'un maillage étatique du territoire équestre. Dans les décennies suivantes, les différents ressorts des haras, qui se sont multipliés, « se dessinaient au coup par coup, au gré des lettres du ministre aux chefs de dépôt. Il faut par ailleurs établir une distinction entre la circonscription du dépôt et la sphère d'influence réelle de l'établissement » (Souvré, 2014). Peu à peu, au cours du XIXe siècle et au fur et à mesure des créations et des fermetures de dépôts d'étalons, une carte plus précise des villes dédiées par l'État à la reproduction équestre se dessine (carte 1). Des villes ayant accueilli des haras royaux d'Ancien Régime (Besançon à partir de 1752, les Rosières-aux-Salines depuis 1768) se voient confirmées sous le Premier Empire mais dans un statut secondaire. Une seconde vague de créations d'importants dépôts d'étalons, décidées ou réalisées durant les années 1840, annonce l'implantation de haras nationaux dans certaines villes (La Roche-sur-Yon, Saintes, Villeneuve-sur-Lot, Hennebont). Le Second Empire est aussi une période de renforcement du rôle de la cavalerie : l'annexion de la Savoie sarde à la France en 1860 promeut ainsi le dépôt d'Annecy. La ville de Compiègne était envisagée dès 1851 comme haras impérial mais la présence de la cour empêche alors l'installation des activités d'étalonnage dans les anciennes Grandes Écuries du Roi. Au final, de nombreux dépôts d'étalons créés après 1806 comme appui aux haras impériaux (Saint-Lô, Tarbes, Cluny, Rodez, Montier-en-Der, Blois, Lamballe) deviennent des haras nationaux avec la loi Bocher de 1874 au début de la IIIe République.

En effet, celle-ci marque un deuxième moment majeur dans l'histoire de ces villes en créant une administration supérieure des haras. La loi Bocher instaure un nouveau découpage du territoire équestre national avec 22 haras nationaux (carte 1) : des circonscriptions, plus petites, s'adaptent désormais sensiblement mieux aux races et aux principales régions d'élevage notamment dans le Sud-Ouest et le Nord-Ouest de la France. C'est ainsi que PauGelos et Libourne n'englobent que deux départements chacun tandis que Lamballe et Saint-Lô seulement un département et demi. Au contraire, le Sud-Est est délaissé à travers une vaste circonscription de 10 départements (dé)centrée sur Perpignan. D'une part, la volonté de choisir des villes centrales dans leur circonscription comme Besançon ${ }^{2}$, Blois, Cluny ou la Roche-sur-Yon, est patente. D'autre part, des haras nationaux se situent dans des villes plutôt en périphérie de leur circonscription : Lamballe et Hennebont reflètent le poids du peuplement de l'Armor breton ; le «Versailles lorrain », Rosières-aux-Salines (à quelques kilomètres de Nancy), se retrouve depuis la fin de la guerre de 1870 à une vingtaine de kilomètres de la nouvelle frontière ; quant à Annecy, sa proximité avec la frontière provient de son rattachement récent à la France. L'incongruité la plus frappante est la faible distance entre les haras nationaux de Pau-Gelos et de Tarbes, une quarantaine de kilomètres : là encore, le rattachement tardif à la couronne française et des terres d'élevage différentes éclairent la situation (Guillotel, 1985) ${ }^{3}$. La loi Bocher a dû trouver un savant équilibre entre centralité

\footnotetext{
2 Alors que précédemment, depuis 1835, le dépôt était installé à Jussey tout au nord de la Haute-Saône (Guillotel, 1985).

Tout comme Pau, Tarbes est assez excentrée par rapport au reste de la circonscription, en étant également un autre « élevage de l'Adour » : celui de la plaine, quand le premier était celui de l'estuaire.
} 
géographique et contraintes politiques ${ }^{4}$. Les haras nationaux ont ainsi été fréquemment établis dans des villes aux fonctions administratives importantes : en 1875, rares sont celles qui ne sont ni des préfectures ni même des sous-préfectures (7 sur 22). "Loin de chercher à implanter les haras au cœur des campagnes productrices, on les isolera dans les grandes villes, en les transformant en édifices publics, à l'instar des préfectures, des casernes ou des lycées » (Guillotel, 1985). De fait, de nombreux dépôts d'étalons prennent place, à un moment ou à un autre, dans des bâtiments religieux réquisitionnés au moment de la Révolution Française. C'est ainsi que « les étalons et les chevaux de l'État ont sauvé de la destruction nombre de nos abbayes, monastères, églises, couvents et quelques autres établissements plus récents dédiés à ces seules activités » (de Sainte Marie, 2012). L'appartenance physique et symbolique de ces haras des (centres)-villes à un tangible quartier du pouvoir public se ressent notamment à Annecy, Blois, Compiègne, la Roche-sur-Yon et Tarbes où l'on trouve la (sous)-préfecture, la gendarmerie, la caserne, le palais de justice ou la prison quasiment accolés aux haras nationaux (carte 1) $)^{5}$. Les haras nationaux participent ainsi à la formation de citadelles de l'État central au cœur de régions parfois longtemps hostiles comme à la Roche-sur-Yon (anciennement nommée Napoléon-Vendée).

La dernière étape de la constitution des villes des haras nationaux correspond au début des années 1970. En 1971, un décret tente d'harmoniser les sphères d'influence des haras avec les circonscriptions d'action régionale (carte 2). Être en phase avec la nouvelle géographie hippique $^{6}$ est aussi un objectif de cette réorganisation : 1'Île-de-France et le Midi, longtemps délaissé, sont de grands territoires de développent hippique, spécifiquement dans le cadre d'une équitation de loisirs. En 1972-1973, le nombre de haras nationaux est porté à 23 avec notamment l'installation de haras aux Bréviaires et à Uzès ${ }^{7}$. Le premier dessert l' ̂lle-de-France et permet au haras national du Pin de se recentrer sur le berceau normand. Dans le second choix, le poids de la centralité est là encore prégnant car l'implantation dans cette ville se fait au détriment d'Arles, d'Ajaccio - qui avaient accueilli précédemment des dépôts - ou de Perpignan. L'absence de haras national pour l'arc méditerranéen depuis 1927 est dès lors comblée ce qui fige la liste des villes des haras nationaux pour une trentaine d'années, jusqu'aux profonds bouleversements que connaît l'État français au début du XXIe siècle.

\section{Les villes des haras nationaux face aux réformes de l'État}

Depuis les années 2000, les missions de service public ainsi que le patrimoine animal, humain et immobilier attribués aux haras nationaux sont inclus dans un mouvement plus général de réforme de l'État. Marqué, entre autres, par une volonté de rationalisation financière et la recherche d'une meilleure efficacité, il occasionne des transformations rapides et profondes des territoires auxquelles les villes des haras nationaux n'échappent pas.

Une première refonte de la carte des haras nationaux est organisée par le premier contrat d'objectifs signés avec l'État pour la période 2003-2008. Elle prévoit le passage de 23 haras nationaux à 20 pôles hippiques ce qui amène la fermeture en 2005-2006 des sites anciens d'Annecy, de Strasbourg et de Blois (carte 2), compensée de manière très incomplète par des transferts d'activités dans des centres techniques respectivement à Chazey-sur-Ain, Pfaffenhoffen et Amboise. La fin du bail emphytéotique des locaux auprès de la ville de

\footnotetext{
$4 \quad$ Ces aspects de représentations identitaires et spatiales du pouvoir étatique sont communs aux processus d'érection des capitales d'État des États-Unis (Montès, 2014).

$5 \quad$ Ce sont toujours les repères urbains d'aujourd'hui selon N. Commerçon (1999).

6 La fermeture du haras national de Libourne en 1955 annonçait déjà ce rééquilibrage territorial en atténuant la prédominance du Sud-Ouest.

$7 \quad$ Cette création a permis de combler ce manque et de ramener la circonscription d'Annecy à des proportions plus équilibrées (Guillotel, 1986) en lui ôtant les Hautes et Basses-Alpes, les Alpes-Maritimes et le Vaucluse ainsi que de soulager celles de Rodez et de Tarbes en détachant notamment la Corse de cette dernière.
} 
Strasbourg a alors été une opportunité. En parallèle, des audits de modernisation sont réalisés, c'est notamment l'objet du rapport Bourdin remis en 2006. Ils insistent sur la nécessaire fermeture d'un certain nombre de pôles hippiques en raison de leur dispersion géographique, sur le pesant entretien d'un patrimoine immobilier à valoriser mais aussi sur l'indispensable recherche de partenariats avec les collectivités territoriales. Ceci annonce une accélération de la modernisation de l'État qui, à partir de 2007, passe par la Révision Générale des Politiques Publiques. Cette RGPP amène une réorganisation des services publics axée sur un désengagement de l'État et un transfert de missions et de compétences aux collectivités territoriales (régions, départements, intercommunalités, communes). En ce qui concerne la réforme des haras nationaux, il est répété que « la valorisation de ce patrimoine relève avant tout de la collectivité territoriale sur laquelle il est implanté » (Bourdin, 2008). C'est ainsi que la loi de finances de 2008 préconise la vente des biens immobiliers pour compenser la baisse des subventions de l'État, ce qui ne va pas sans provoquer des craintes dans l'ensemble de la filière. Dans le cadre du second contrat d'objectifs 2009-2013, les rumeurs prolifèrent sur PauGelos $^{8}$ et Tarbes $^{9}$ par exemple mais surtout sur Compiègne après sa fermeture partielle au public en 2008 (photographie 1). L'administration des haras nationaux fusionne alors avec l'École Nationale d'Équitation de Saumur (surnommée «le Cadre noir ») pour former l'Institut français du cheval et de l'équitation (Ifce) depuis début 2010. La volonté d'améliorer l'efficacité de l'administration tout en réalisant une publicité systématique autour des mesures de réforme de l'État apparaissent comme un véritable « souci de soi de l'État» (Bezes, 2009a).

Cette politique revendiquée de " résultats » visibles (Bezes, 2009b) du repli étatique menace d'autant plus les villes des haras nationaux qu'elles sont, pour une grande majorité d'entre elles, des villes petites et moyennes (carte 2). Or ces villes comportent des fonctions administratives et politiques qui « ont subi de plein fouet la refonte de l'organisation territoriale de l'État » (Demazière, 2014). Elles sont d'autant plus sensibles à la question du maintien des services publics que ceux-ci représentent souvent le fondement traditionnel de leur rôle d'animation des territoires (Édouard, 2012). Certaines de ces petites et moyennes villes des haras nationaux ont été atteintes par la reconfiguration d'autres schémas territoriaux administratifs avec pour corollaire des pertes d'emplois publics dans la Justice (Tribunal de Commerce de Villeneuve-sur-Lot en 2008, Tribunal d'Instance de Saint-Lô en 2009), dans le Trésor (La Roche-sur-Yon en 2009, Annecy en 2010), l'Armée (Compiègne en 2012) ou la Santé (Hennebont, Rodez, Tarbes, Villeneuve-sur-Lot). Un effet boule de neige des réformes en matière de services au public se met alors en place (Taulelle, 2012). Par ailleurs, la Modernisation de l'Action Publique (MPA) a pris le relais en 2012 de la RGPP et a mis en question une quarantaine de petites sous-préfectures, dont celle de Saintes, dans le cadre des nouvelles régions et de la redéfinition du rôle des départements ${ }^{10}$.

La persistance du désengagement de l'État se retrouve dans l'actuel contrat d'objectifs (2014-2017) qui prévoit que " faute d'accord ou de partenariat [avec les collectivités territoriales ou des partenaires privés] au 31 décembre 2015, les sites qui ne sont pas à vocation nationale et dont l'Ifce est propriétaire, devront être vendus ». La date butoir approchant, 10 haras nationaux sont annoncés à la vente pour 2016 (carte 2), ce qui entraîne une mobilisation locale variée. En effet, le sentiment d'un « aménagement menacé » (Subra,

\footnotetext{
$8 \quad$ Question n 33235 de Mme Lignières-Cassou Martine publiée au Journal Officiel le 21 octobre 2008.

9 Question n ${ }^{\circ} 405$ de Mme Robin-Rodrigo Chantal publiée au Journal Officiel le 2 décembre 2008.

10 Les mois d'août et de septembre 2015 ont été l'occasion, pour le ministre de l'Intérieur Bernard Cazeneuve, d'affirmer la volonté de transformer de nombreuses sous-préfectures en « Maison de l'État » en les mutualisant avec diverses directions départementales dans le cadre d'une réforme préfectorale.
} 
2007) a parfois été relayé par des élus, particulièrement des sénateurs ${ }^{11}$, ou des articles dans des quotidiens régionaux. A chaque menace de fermeture depuis les années 2000, les inquiétudes et la volonté de freiner la réforme de l'État sont reprises avec un fort impact par ces journaux que ce soit pour Les Bréviaires (Le Parisien), Saint-Lô (Ouest-France), Rodez (La Dépêche du Midi) ou Saintes (Sud-Ouest). Cette refonte de la carte des haras est aussi le marqueur du renforcement de la quantification d'un patrimoine humain, animal et immobilier. En effet, des indicateurs quantitatifs apparaissent dans les contrats d'objectifs (nombre de jours de formation, de stages, coût salarial par site, dépenses de fonctionnement...), les villes des haras nationaux sont ainsi de plus en plus dépendantes d'un management étatique par objectif et d'un financement à la performance (Eyraud, 2011). Cette nécessité de la valorisation est évidente en ce que l'organisme chargé de la vente des sites à partir de l'été 2016 au nom de l'Ifce est la Sovafim (Société de valorisation foncière et immobilière) dont l'État est l'unique actionnaire. Les bâtiments des haras sont souvent décrits comme coûteux à entretenir de par leur ancienneté, leur emplacement et leur surdimensionnement pour des besoins de moins en moins importants. Dès lors, l'État instaure une forme de pression dans le dernier trimestre de 2015 sur les collectivités territoriales pour qu'elles reprennent l'activité de ce qui reste des haras nationaux installés dans des villes de l'entre-deux, fragilisées au cœur de leurs fonctions.

\section{Les haras nationaux dans les villes : un passé encombrant ?}

Les chemins empruntés par les villes des haras nationaux sont très divers : ils dépendent à la fois de la mobilisation (politique, financière, touristique, médiatique...) des collectivités territoriales, du poids démographique des villes des haras nationaux, de la place de la fonction équine par rapport aux autres fonctions urbaines mais aussi de l'emplacement, d'un éventuel classement aux Monuments Historiques (carte 2) et de la superficie de cette institution dans le tissu urbain local (tableau).

Héberger une prestigieuse fonction équestre dans un espace central restreint peut paradoxalement devenir une entrave: enclavé dans une ville de taille moyenne, le haras national subit un manque de place pour ses écuries, ses pistes et ses laboratoires ainsi que des nuisances urbaines (bruit, pollution...). Ce besoin d'espace a pu se faire ressentir dès la loi Bocher de 1874 car un effort d'amélioration des bâtiments des haras nationaux est alors lancé. Dans les années 1880, les haras nationaux de Blois et Annecy sont reconstruits, dans un plan analogue plus aéré ${ }^{12}$, toujours dans le centre-ville. Cette question de la difficile centralité dans une ville moyenne se poursuit dans une seconde vague un siècle plus tard avec les haras d'Angers et d'Aurillac, parmi les «plus mal implantés de France » (Guillotel, 1986). Tout au long du XXe siècle, l'enceinte du premier a souffert de la pression urbaine et de la proximité de la gare ainsi que des voies ferrées sur sa façade Sud, gênant par le fait l'accès et l'exercice des chevaux. En 1974, le haras est transféré dans un domaine de la périphérie du Lion d'Angers, jusqu'ici principale station de monte dépendante d'Angers. Les mêmes problèmes touchaient le haras enserré dans le tissu urbain central d'Aurillac : en 1983, il est installé dans la couronne périurbaine.

Au-delà des reconstructions sur place et des déménagements ${ }^{13}$, il est frappant de voir que les fermetures précoces de haras nationaux ont lieu dans les villes les plus peuplées : Annecy,

\footnotetext{
11 Ainsi en est-il de Jean-Pierre Bel en 2004 et d'Anne-Marie Escoffier en 2009 pour le site de Rodez, respectivement les questions $\mathrm{n}^{\circ} 12961$ et $0385 \mathrm{~S}$ au ministre de l'Agriculture.

12 «Axe de symétrie avec des bâtiments autour de la carrière centrale, même élévation des façade » (Kinossian, 2012).

$13 \quad$ Il est à noter que l'enserrement urbain, la vétusté des locaux et les visées immobilières sont du même ordre dans l'évolution de la géographie des prisons (Milhaud, 2009) et des casernes urbaines.
} 
Blois et Strasbourg comportent plus de 40000 habitants (tableau). Ces fermetures sont liées à la polyvalence fonctionnelle : les haras nationaux ne sont qu'un service public parmi d'autres, une activité peu essentielle face aux volontés de revitalisation culturelle d'un quartier de ville ou d'un potentiel immobilier de bâtiments au cœur d'un marché très tendu dans les grandes villes. De fait, l'emplacement classé et restreint, moins de 5 hectares, occupé par ces haras nationaux de centre-ville peut devenir un lieu d'enjeux et de développements urbanistiques. Ceci est accentué quand il s'agit d'anciennes implantations monastiques qui, initialement en marge de la ville médiévale, ont connu des réaffectations variées (casernes, hôpitaux, collèges...) et peuvent représenter jusqu'à nos jours d'importantes réserves foncières pour les villes (Andrieux, 2014). Par exemple, l'espace libéré par les haras nationaux dans un ancien couvent au cœur d'Aurillac est aujourd'hui occupé par un centre culturel. Le site de Strasbourg accueille dorénavant une annexe de l'Institut de recherche contre les cancers de l'appareil digestif (Ircad) dans un cadre rénové de 2010 à 2013 avec brasserie et hôtel de luxe. Les anciens locaux des haras nationaux de Blois ont été vendus en janvier 2011 à un promoteur immobilier qui prévoit là aussi restauration, hôtel de charme et salle de congrès. Quant à Annecy, et ce malgré le réaménagement de la fin du XIXe siècle, le haras national est repéré dès 2004 comme occupant un " site très enclavé que la ville est prête à reprendre pour une autre utilisation »(Bourdin, 2008). Le haras national, un des symboles du pouvoir dans la ville, est alors au coeur d'un conflit d'acquisition et d'usage entre propriétaire, investisseurs, associations et élus (Kinossian, 2012). Depuis cette période de tensions, la ville a préempté l'espace concerné, a percé une nouvelle rue à travers le quartier et installé une scène nationale de théâtre depuis 2012. Dans ces cas de sites classés, leur écrin patrimonial a constitué une chance dans leur reconversion fonctionnelle.

Ces problématiques n'épargnent pas les autres villes moyennes des haras nationaux. Parmi les sites en sursis à la fin de l'année 2015 (carte 2), 6 haras paraissent fortement menacés de fermeture en 2016 et forment une catégorie spécifique. Il s'agit de petits haras nationaux de moins d'une dizaine d'hectares, alors que la moyenne se situe autour d'une trentaine, plutôt établis en centre-ville. Leur fermeture avait déjà été évoquée à plusieurs reprises depuis les années 2000 (tableau). En effet, l'activité semble difficile à faire perdurer : entre autres, ces villes n'ont obtenues qu'un rang très secondaire (niveau 1, simple initiation) d'école d'attelage labellisée «Haras National », une forme de compensation à la fin de l'étalonnage par l'accueil de formations rémunératrices. De plus, ces villes de taille moyenne ne disposent pas de sites internet dédiés à leur haras national (hors Ifce), ce qui révèle le manque d'intérêt pour la fonction équine. En dépit, ou en raison, d'un site récent (1983) spacieux accessible et à l'architecture fonctionnelle, le haras national d'Aurillac ne mobilise pas les foules. Situé en périphérie entre l'hippodrome et l'aérodrome, son éventuelle fermeture n'étonne pas en ce qu'elle ne porterait pas atteinte au prestige de la ville. De l'aveu du délégué de la vingtaine d'employés, « on va vers une vente certainement morcelée du site. Car qui pourrait acheter un tel ensemble ? »(Rabhi, 2015). Son déficit médiatique est telle que plusieurs articles journalistiques évoquant les éventuelles fermetures mentionnent, par une faute d'orthographe peut-être issue de la dépêche AFP originelle, un haras national à Auriac en Corrèze... De manière générale, la mobilisation des collectivités territoriales pour éviter les fermetures est insuffisante ou infructueuse : par exemple, un litige opposant la mairie de Villeneuve-sur-Lot et l'Etat sur la propriété des terrains ne fait que retarder la mise en vente par la Sovafim. Sans soutien d'une autre collectivité territoriale, les communes et agglomérations ne sont pas toujours suffisamment armés pour supporter le poids financier et immobilier d'un haras d'envergure nationale. Les 9 hectares installés dans le centre-ville de Tarbes sont hors de prix pour une ville de cette taille, sauf si l'Etat privilégie l'identité équestre à une plus-value immobilière. 
Inversement, l'enracinement des haras nationaux est beaucoup plus important dans 11 villes avec une forte implication des conseils départementaux qui sont, pour 8 d'entre eux, au moins les copropriétaires des murs voire les principaux gestionnaires. De surcroît, ils sont en première ligne pour racheter les haras de Saint-Lô ${ }^{14}$ et de Lamballe $^{15}$ (photographie 2) ou contribuer financièrement à la reconversion du site de Compiègne en musée. Sans surprise, les villes de l'Ouest de la France sont surreprésentées : la ville d'Hennebont et l'agglomération lorientaise cherchent à racheter le haras morbihannais et même si une entreprise privée s'est installée au Lion d'Angers ${ }^{16}$ les bâtiments appartiennent au Maine-et-Loire depuis le transfert de 1974. Effectivement, ce sont les Conseils départementaux qui ont saisi l'opportunité de s'approprier l'atout économique délaissé par l'État à partir des années 2000. Le haras national des Rosières-aux-Salines est devenu paradoxalement « régional » en tombant dans l'escarcelle du Conseil départemental de Meurthe-et-Moselle. Autour du site bourguignon s'est forgé une "Équivallée Cluny », soutenue par le Conseil régional, le Conseil départemental et la commune, comprenant aussi un centre équestre et l'hippodrome tout en profitant du site classé de l'abbaye. Au contraire des projets de revitalisation immobilière et culturelle dans les villes de strate supérieure, les municipalités plus petites se sont efforcées de maintenir l'activité équine au cœur de leur territoire, relayant ainsi une forte demande sociale de continuité des traditions locales. Pour défendre le haras de Saint-Lô, les élus ont mis en avant les travaux déjà engagés à hauteur de 15 millions d'euros pour entretenir des bâtiments reconstruits à l'identique après les bombardements de 1944 ainsi que l'inscription aux Monuments historiques depuis 1993. La structuration urbaine de la fonction équine y est telle (centres d'entraînement quasiment autofinancés, participation aux Jeux Équestres Mondiaux...) que la réponse apaisante du Ministre de l'Agriculture Stéphane le Foll fut assez rapide : « si le haras était vendu, ce serait au Syndicat mixte »(Baillet, 2015). L'État juge ainsi les collectivités territoriales dignes de le suppléer, voire de réaliser un service public plus efficace (Blot, 2012), même si cela doit passer, au vue des fermetures annoncées, par un reflux sur des régions où l'identité équine est traditionnelle. De plus, les collectivités territoriales insistent sur le cadre paysager des haras nationaux : la formule réitérée d'un «écrin de verdure en plein centre-ville » montre bien que ces villes veulent conserver ce qui est ressenti comme précieux dans un contexte de marketing urbain très concurrentiel. Les haras nationaux émergent alors comme un atout touristique ${ }^{17}$ mis en avant par les petites et moyennes villes dans leurs rapports avec les grandes métropoles qui accaparent les fonctions tertiaires supérieures. Cette stratégie patrimoniale peut s'inscrire dans le cadre départemental : organiser des animations équestres au cœur du territoire vendéen permet à l'ancien haras national de la Roche-sur-Yon, devenu symptomatiquement « Haras de la Vendée » depuis 2008 (photographie 3), d'esquisser un rééquilibrage du territoire face au poids démesuré du tourisme côtier. Les sites internet consacrés et l'installation d'école d'attelage de niveaux 1 et 2 (tableau) confirment la mobilisation réussie des collectivités territoriales en faveur de certaines villes des haras nationaux pour qui c'est une question de survie économique.

Enfin, là où certaines villes moyennes laissent s'effacer de leur tissu urbain l'ancienne fonction équine et que d'autres se mobilisent pour la valoriser, d'autres villes des haras

14 Un syndicat mixte constitué pour moitié de la région Basse-Normandie et du département de la Manche gère un pôle hippique formé autour du haras depuis 2005.

Un syndicat mixte constitué de la région Bretagne, du département des Côtes d'Armor, de Lamballe communauté et de la ville de Lamballe gère le haras depuis 2006.

${ }_{16}$ Ce haras est implanté à la limite méridionale de la Mayenne, département qui, depuis plusieurs décennies et grâce à un foncier accessible, constitue un prolongement majeur du berceau normand. Cette émergence se reflète dans un logo départemental très équestre.

17 La Fédération des Maires de Villes Moyennes (FMVM) présentait dans un colloque de 2010 le tourisme comme « une opportunité pour dynamiser les villes moyennes » et plus largement leur territoire (Delaplace, 2013). 
nationaux en ont fait leur emblème. Ainsi, les monumentaux haras du Pin et de Pompadour ${ }^{18}$ sont établis depuis la fin du XVIIIe siècle dans de petites communes de quelques centaines d'habitants et celui d'Uzès peut aussi être qualifié de haras des champs en ce qu'il est localisé en lisère d'une petite ville de moins de 9000 habitants (tableau). Par le fait, leurs problématiques sont très différentes des haras nationaux précédents : loin du centre-ville, ils ne subissent pas de véritable pression urbaine. Ce sont même les haras nationaux qui ont fait se développer ces «villes » comme à Arnac-Pompadour où la commune se résume au château, aux haras et à l'hippodrome ${ }^{19}$. Cette autoproclamée « cité du cheval » comptant environ 80 employés rayonne en plusieurs domaines dispersés dans les hameaux des alentours (Le Monde, 2015). Les haras nationaux du Pin et d'Uzès accueillent des écoles d'attelage d'ampleur nationale (niveau 3). En plus d'un service public rare, le statut de haras national a apporté à ces communes une certaine stabilité économique. Les ressources humaines engendrées, certes modestes avec quelques dizaines d'employés, restent essentielles dans ce type de structure urbaine. Lorsque des rumeurs (Le Figaro, 2011) ont évoqué la fermeture et la vente du vaisseau-amiral des haras nationaux - Le Pin-au-Haras est surnommé le « Versailles du cheval »-, toute une région d'élevage ${ }^{20}$ s'est mobilisée pour maintenir en vie de bâtiments historiques et majestueux mais aussi très coûteux à entretenir. Reflet d'une mondialisation du monde hippique, « la rumeur d'une reprise par des Qataris a couru » alors qu'il s'agit du troisième pôle touristique de Basse-Normandie après le Mont-Saint-Michel et le Mémorial de Caen (Libération, 2012) et d'une composante essentielle des Jeux Équestres Mondiaux de 2014. Un Établissement Public Administratif a été créé en 2015 pour être géré conjointement par le département (dont le logo est composé d'un cheval), la région et l'État à travers l'Ifce et pour financer les lourds travaux de rénovation estimés à 50 millions d'euros. Au-delà des enjeux symboliques, urbanistiques et fonciers, les nombreuses contraintes architecturales encadrées par le Ministère de la Culture et ses représentants locaux (Direction Régionale des Affaires Culturelles, Architectes des Bâtiments de France, Conservateur Régional des Monuments Historiques...) peuvent être un frein à ce type d'investissement exceptionnel. Au contraire des fermetures annoncées en 2015-2016, ces 3 haras ont ainsi été confortés par l'Ifce, qui en reste l'unique ou le principal propriétaire, comme des vitrines nationales de la filière équine avec des ressorts assez évidents : le Nord-Ouest pour Le Pin, le Sud-Ouest pour Pompadour et le Sud-Est pour Uzès.

$\mathrm{Au}$ final, quelle que soit la direction prise, les haras nationaux participent aux transformations des usages de leur ville d'accueil. Les villes se sont diversement appropriées les haras nationaux tout comme ceux-ci ont été un ferment essentiel pour certaines d'entre elles (anciens bâtiments religieux, quartier public...). Cependant, les interrogations restent entières sur les capacités de résilience des villes de l'entre-deux accueillant encore un haras national.

\section{Conclusion}

Selle français, percheron, breton... : les races de chevaux et leur berceau territorial respectif ont été déterminants dans la localisation première des villes haras nationaux au XIXe siècle. Les années 1960-70 ont été l'occasion d'un certain renouveau des haras nationaux dès

\footnotetext{
18 Ces deux haras ont participé à l'émission télévisuelle de France 2 sur les monuments préférés des Français respectivement en 2014 et 2015.

19 Des hippodromes se situent systématiquement à proximité des villes des haras nationaux voire dans la même, plutôt petite, commune pour 13 d'entre eux (Bruneau, 2014), formant ainsi un véritable système équestre.

20 Entre les haras, les hippodromes et les centres équestres, la pression foncière y est particulièrement forte, d'autant plus que le haras national du Pin est installé sur plusieurs centaines d'hectares suscitant beaucoup d'envies.
} 
lors en phase avec le découpage du territoire français en régions. Ce réseau de villes des haras nationaux a été longtemps relativement invisible dans le paysage administratif français, sauf quand il s'agit de le supprimer notamment pour des raisons financières. Dès lors, la réforme de l'État amène une rupture car elle touche d'autant plus ces villes qu'elles sont ancrées dans des départements aux fonctions en pleine redéfinition : l'impact économique, social et financier des réformes récentes reste encore à mesurer.

De plus, les haras nationaux participent à une mise en compétition des villes et des espaces intra-urbains de par l'implantation fréquemment centrale et prestigieuse de la fonction équine. Par conséquent, leur devenir est très varié et dépend notamment de l'enracinement de la filière dans la ville mais aussi dans l'espace environnant. C'est ainsi que certaines grandes villes des haras nationaux se défont de leur fonction équine pour désormais en valoriser les façades immobilières ainsi qu'un emplacement avec d'importantes potentialités. D'autres petites villes, au contraire, se voient conforter dans leur rôle d'étendard historique et patrimonial de la filière : l'essor d'écoles d'attelage officielles, dans un cadre plus que légitime, souligne une tentative de réponse au renouveau des besoins en traction animale. Entre ces deux extrémités, l'évolution de la plupart des villes des haras nationaux dépend des capacités de mobilisation des collectivités territoriales, plus fortes dans les régions traditionnelles d'élevage (Normandie, Pays de la Loire), et de leurs stratégies de développement urbain. Dès lors, on assiste, quand cela est possible, à une mise en tourisme des haras nationaux et ceci sur l'année entière (spectacles estivaux, marchés de Noël...). L'installation de l'office de tourisme de Lamballe à l'intérieur du haras national ainsi que celle du Grand Départ du Tour de France 2016 dans celui de Saint-Lô sont révélatrices des tendances de ces dernières années.

En définitive, les haras nationaux survivants, qui ont fait et font encore la ville, apparaissent comme le réceptacle d'un passé glorieux. Repris en main à l'échelle locale, les haras nationaux sont les témoins parfois gênants d'une époque où ils représentaient un prestigieux joyau de l'État au cœur des villes et non un patrimoine urbanistique et foncier à céder pour se désendetter.

\section{Références bibliographiques :}

Articles scientifiques :

ANDRIEUX, J.-Y. (dir.) 2014. Villes de Bretagne, patrimoine et histoire. Rennes, PUR.

BEZES, P. 2009a. Réinventer l'État. Les réformes de l'administration française (19622008), Presses universitaires de France, coll. « le lien social », Paris.

BEZES, P. 2009b. «Les rationalités politiques dans la réforme de l'État: le cas de la V République », Revue d'histoire moderne et contemporaine $\mathrm{n}^{\circ}$ 56-4bis, p. 54-74.

BLOT, P. ; COURCELLE, T. 2012 «'Bien observer sans se faire remarquer', est-ce toujours possible avec la RGPP ? Le rôle social et l'implantation territoriale de la gendarmerie en question », in TAULELLE, F. (dir.) « Le délaissement du territoire. Quelles adaptations des services publics dans les territoires ruraux ? », Sciences de la société n ${ }^{\circ} 86$, p. 14-33.

BOURDIN, J. 2008. Les Haras nationaux. Pour un outil modernisé au service de la filière cheval, rapport d'information fait au nom de la Commission des Finances, Paris.

BRUNEAU, D. 2014. «Les hippodromes au rythme des saisons: localisation et intensité des courses hippiques », Mappemonde $\mathrm{n}^{\circ} 113$.

COMMERÇON, N. 1999. «Emploi public et logiques territoriales aux marges d'un espace métropolisé », Revue de géographie de Lyon n74/2, p. 141-151.

DELAPLACE, M. 2013. "Le tourisme dans les villes moyennes : vers des politiques coordonnées », Métropolitiques. 
DEMAZIÈRE, C. 2014. "Pourquoi et comment analyser les villes moyennes ? Un potentiel pour la recherche urbaine », Métropolitiques.

DIACT 2005. Les villes moyennes françaises. Enjeux et perspectives. Collection Travaux.

ÉDOUARD, J.-C. 2012. « La place de la petite ville dans la recherche géographique en France : de la simple monographie au territoire témoin $»$, Annales de géographie $\mathrm{n}^{\circ} 683$, p. $25-$ 42.

EYRAUD, C. ; EL MIRI, M.; PEREZ, P. 2011. «Les enjeux de quantification dans la LOLF. Le cas de l'enseignement supérieur. », Revue Française de Socio-Économie $\mathrm{n}^{\circ} 7$, p. 147-168.

FLOCH, J.-M. ; MOREL, B. 2011. Panorama des villes moyennes, Direction de la Diffusion et de l'Action Régionale, Insee.

GUILLOTEL, G. 1985-1986. Les Haras nationaux (3 vol.), Paris, C. Lavauzelle.

KINOSSIAN, Y. 2012. "Les haras dans la ville d'Annecy. Hier, aujourd'hui, demain, $\mathrm{XIX}^{\mathrm{e}}-\mathrm{XXI}^{\mathrm{e}}$ siècle », In Situ.

MILHAUD, O. 2009. Séparer et punir - Les prisons françaises : mise à distance et punition par l'espace, Thèse de doctorat de géographie.

MONTÈS, C. 2014 American Capitals. A Historical Geography, The University of Chicago Press, Chicago.

MULLIEZ, J. 2004. Les chevaux du royaume : aux origines des haras nationaux, Paris, Belin.

(de) SAINTE MARIE, T. 2012. "Abbayes, couvents et monastères devenus dépôts d'étalons impériaux, royaux et nationaux », In Situ.

SANTAMARIA, F. 2000. " La notion de 'ville moyenne' en France, en Espagne et au Royaume-Uni », Annales de Géographie nº13, p. 227-239.

SANTAMARIA, F. 2012. «Les villes moyennes françaises et leur rôle en matière d'aménagement du territoire : vers de nouvelles perspectives ?», Norois n`223, p. 13-30.

SOUVRÉ, A. 2014. L'œuvre des haras, entre amélioration animale et création architecturale. L'implantation du dépôt d'étalons de Cluny (premier quart du XIXe siècle), Thèse de l'Ecole des Chartes.

SUBRA, P. 2007. Géopolitique de l'aménagement du territoire, A. Colin, coll. Perspectives Géopolitiques, Paris.

TAULELLE, F. 2012 «Ce que nous avons vu en matière de services publics dans les quatre pays de l'étude : le délaissement du territoire» in TAULELLE, F. (dir.) « Le délaissement du territoire. Quelles adaptations des services publics dans les territoires ruraux ?», Sciences de la société n86, p. 5-13.

Articles de presse :

BAILLET, Z. 2015. « A Saint-Lô, le haras restera propriété publique », Ouest-France, 7 novembre 2015.

LAUNET, E. (2012). «Le déclin de l'empire équin », Libération, 19 juin 2012.

Le Figaro (2011). « Le Haras du Pin mis en vente », 7 avril 2011.

Le Monde (2015). « Le royaume englouti de l'Anglo-Arabie », 8 janvier 2015.

Le Télégramme (2015). « Hennebont toujours en selle », 24 septembre 2015.

RABHI, C. (2015). «Le haras d'Aurillac, mis en vente ou pas ? », La Montagne, 3 octobre 2015.

$\underline{\text { Sources : }}$

http://www.ifce.fr/

dont les contrat d'objectifs et de performance pour 2014-2017 : 
http://www.ifce.fr/wp-

content/uploads/2015/09/INS_Contrat_d_objectifs_et_de_performance_de_1_Ifce.pdf

http://www.equivallee-cluny.fr/

http://www.haras-hennebont.fr/

http://www.haras-lamballe.com/

http://haras.vendee.fr/ pour la Roche-sur-Yon

http://www.harasdulion.com/ pour Le Lion d'Angers sous régime privé depuis 2014

http://www.haras-national-du-pin.com/

http://www.haras-des-breviaires.com/ pour la partie reproduction privatisée en 2012

http://www.harasderosieres.com/ sur le site des Rosières-aux-Salines désormais de niveau régional sous la forme d'une Société Coopérative d'Intérêt Collectif (Scic) depuis 2012

http://polehippiquestlo.fr/actualites/haras-de-saint-lo-saison-2015.html

http://haras-saintlo.fr/ sur la Société À Responsabilité Limitée (Sarl) chargée de la reproduction

http://www.les-haras.com/historique/ sur les anciens haras nationaux de Strasbourg

http://www.insee.fr/

Recensement de la population 2013 - Limites territoriales au 1er janvier 2015

Titre carte 1 : Les haras nationaux et leurs circonscriptions en 1875

Titre carte 2 : Les haras nationaux et leurs circonscriptions depuis 1974

Titre et légende de la photographie 1: La cour intérieure du haras national de Compiègne La proximité de l'agglomération parisienne ne fait qu'accentuer les tentations immobilières sur un site historique, verdoyant et classé au cœur d'une grande ville moyenne. Après avoir servi lors des Jeux Équestres Mondiaux de 2014, il est l'objet de projets, d'appel à mécènes et de l'élaboration de slogans ( Le haras est en vie, il est en ville, il donne envie !»). Malgré la mobilisation de la ville et du conseil départemental, la vente du site en 2016 ou, au contraire, l'installation d'un musée national des transports hippomobiles dépend du bon vouloir de l'Etat. Une solution transitoire est recherchée pour éviter l'occupation et la dégradation d'un haras fermé au public depuis 2008.

Titre et légende de la photographie 2 : L'accueil du haras national de Lamballe Pendant plusieurs années, le haras a dû faire face aux besoins d'espace du centre hospitalier voisin (derrière les arbres). Peu à peu, la communauté de communes de Lamballe s'approprie le haras national : contrat d'occupation temporaire signé en 2006, la même année installation de l'office de tourisme (à gauche), des agents de Lamballe communauté en 2011, du musée de la ville (Mathurin Méheut) envisagée pour 2018-2019... Le récent classement des bâtiments aux Monuments Historiques en février 2016 vient opportunément empêcher une reconversion en logements.

Titre et légende de la photographie 3 : L'entrée principale du haras de la Vendée L'ancien haras national de la Roche-sur-Yon est devenu un des sites touristiques majeurs du conseil départemental. Au-delà de son appartenance à un pôle étatique fort (préfecture, cité administrative, écoles), l'accès au public est très apprécié de par sa proximité avec les vastes espaces verts du cours Bayard, son intégration dans un « circuit Napoléon » intra-urbain (place centrale avec statue équestre surélevée, lycée, théâtre, hôpitaux...) et l'organisation de spectacles estivaux accueillant plusieurs milliers de personnes à chaque représentation. 